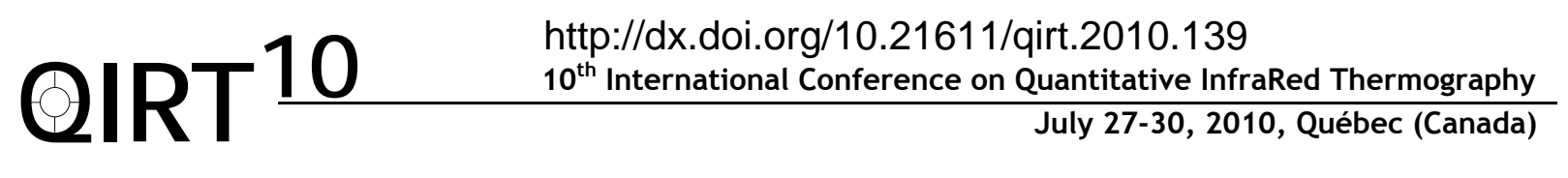

\title{
Pixel-wise real-time calibration method for infrared cameras
}

\author{
by P. Tremblay*, L. Belhumeur*, M. Chamberland*, A. Villemaire*, P. Dubois*, F. Marcotte*, C. Belzile*, \\ V. Farley*, P. Lagueux*
}

*Telops, 100-2600 avenue St-Jean-Baptiste, Québec (Québec), Canada G2E 6J5, pierre.tremblay@telops.com

\begin{abstract}
Accurate radiometric calibration is a key feature of modern infrared cameras. Considering the newly available focal plane arrays exhibiting very high spatial resolutions (>megapixel) and very high readout speeds ( $>1000$ frames per second) along tailored spectral bands, Telops has developed a method to provide a real-time radiometric calibration for every pixel of the array.

The novel approach first accounts for the spatially varying spectral responsivity across focal plane array pixels. The information required for this correction is measured in the laboratory by acquiring the photonic flux versus the temperature of a calibration source for each pixel of the array, with the instrument temperature held constant. It is assumed that these gain factors are stable in time, so this characteristics needs to be measured infrequently.

The method also manages offset effects which are known to vary significantly in time. The offset terms are principally composed of instrument self-emission and thus are strongly correlated with its temperature. First the emission of the camera lens is characterised in the laboratory by measuring the offset changes versus the lens temperature. Second the instrument self-emission is also characterised in the laboratory by measuring the offset changes versus the instrument temperature. Correction in the field of both of these effects is performed in real-time using a lookup table indexed using the instrument and lens temperatures. A validation flag is also available in the field by comparing the temperature of an internal reference and the predicted radiation temperature obtained from our nominal algorithm.

We demonstrate the real-time implementation of this novel method for the pixel rates compatible with the fastest infrared cameras. Experimental results are also presented to illustrate the end-to-end radiometric performance for various scenarios.
\end{abstract}

